\title{
ASPECTS OF THE TERRESTRIAL INFLUX OF SMALL METEOROIDS
}

\author{
A.D. TAYLOR AND W.G. ELFORD \\ Department of Physics and Mathematical Physics \\ University of Adelaide, Adelaide, 5005, South Australia
}

\begin{abstract}
This paper briefly summarises the contribution to the understanding of meteoroids and their orbits from recent radar studies of meteors. New data from VHF and UHF observations indicates that some meteoroids are ablating at heights up to $160 \mathrm{~km}$ and modelling work suggests that such meteoric ionization can only be deposited by meteoroids that ablate at temperatures below $1000^{\circ} \mathrm{K}$. Meteoroids with entry speeds well in excess of $100 \mathrm{~km} / \mathrm{s}$ are clearly interstellar and some tentative sources have been identified. The distributions of the orbital elements of earlier data have been reanalysed in the light of a new understanding of the selection effects in those radar studies, and the presence of a significant set of retrograde orbits identified. The new technique of observing meteors 'coming down the radar beam' has led to very accurate measures of deceleration and detailed analysis of fragmentation events.
\end{abstract}

\section{Introduction}

The bodies that contribute to the meteor phenomenon in the atmosphere are $6-10$ orders of magnitude smaller than most objects that produce impact features on the surface of the planets. Nevertheless, the study of meteors is relevant to any discussion of impacts of large bodies with the planets as the sources of meteoroids are comets and asteroids, and the meteoroids carry some degree of 'memory' of their progenitors. In the case of meteors of cometary origin there is a close association between the orbits of many meteor streams and the orbits of the parent bodies. Further, observations of meteor phenomena over time scales as short as one year lead to significant statistics regarding the distributions of the mass, velocity, ablation characteristics, and orbital elements of these small bodies. Radar and optical (video) observations in recent years have indicated that a significant fraction of meteoroids ablating in the atmosphere have low ablation temperatures, and a small fraction are interstellar. Evidence for these new families of meteoroids is presented below, together with some comments on the way in which radar observations are giving a new understanding of the structure of stony meteoroids.

\section{Meteor heights}

Particles of mass $>10^{-10} \mathrm{~kg}$ with a speed of $20-60 \mathrm{~km} / \mathrm{s}$ ablate in the atmosphere to produce narrow trails of ionization detectable by radar, while the trails formed by more massive particles can be observed with video systems. VHF radars observe meteors up to a height of about $115 \mathrm{~km}$, while MF radars have detected a substantial flux of meteors at heights up to $140 \mathrm{~km}$ (Steel and Elford, 1991). These height limits are determined predominantly by the radio scattering process rather than the actual meteor phenomenon. UHF incoherent scatter observations (EISCAT, $930 \mathrm{MHz}$ ) have detected short term enhancements in the level of ionization in a $1 \mathrm{~km}$ cube of atmosphere at $160 \mathrm{~km}$ that have been attributed to meteors (Pellinen-Wannberg and Wannberg, 1996). These observations imply that conventional meteor radars operating in the frequency range $30-50 \mathrm{MHz}$ are observing only a fraction of the meteors occurring in the atmosphere. This conclusion is supported by a comparison of meteoroid fluxes inferred from radar observations and those determined from impacts on spacecraft, which suggests that about $90 \%$ of meteors are not detected by radar. 

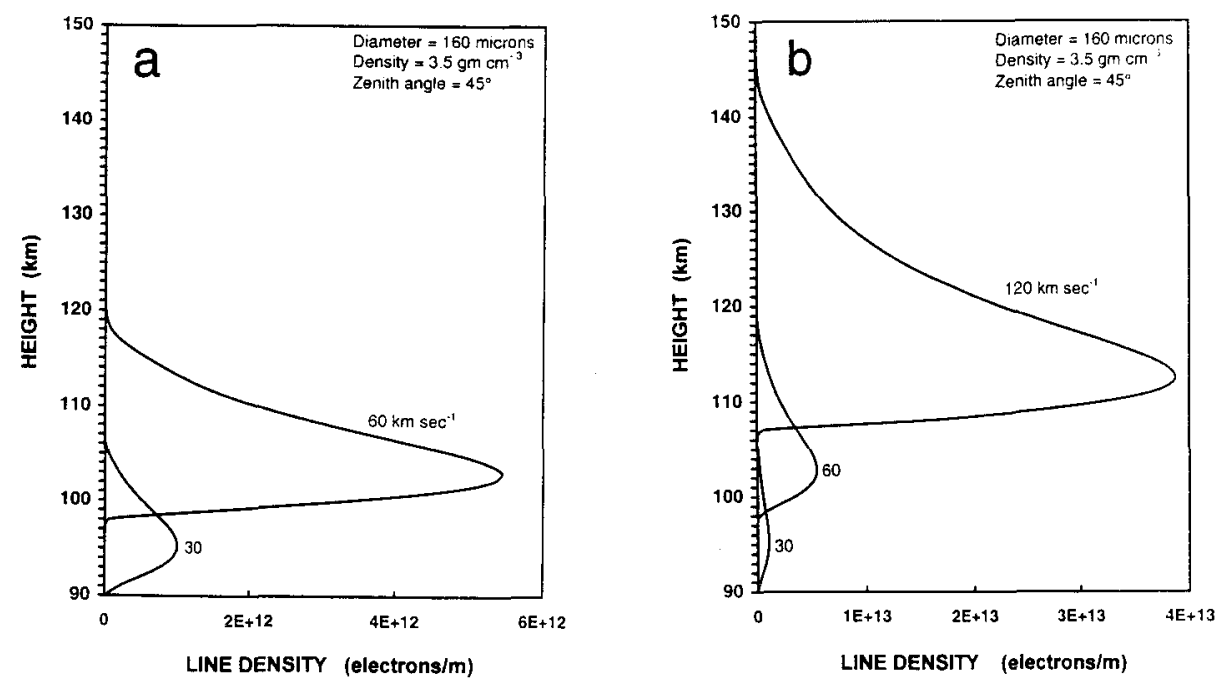

Figure 1. Effect of the incident velocity on the ionisation profile of a stony meteoroid

\section{Ablation Characteristics of Meteoroids}

The heights at which stony meteoroids ablate can be estimated by applying some well established analysis procedures to small particles entering the atmosphere, being heated by collisions with air molecules and subsequently evaporating. The most comprehensive investigation is that of Love and Brownlee (1991) although the heating rate they determine is too fast due to an incorrect choice of specific heat for stony particles. Love and Brownlee used a value of $10^{6} \mathrm{ergs} / \mathrm{g} \cdot \mathrm{K}$ which they attributed to Öpik (1958). The actual value given by Öpik (Appendix 2, p. 161) for 'meteoritic stone' is $9 \times 10^{6} \mathrm{ergs} / \mathrm{g} \cdot \mathrm{K}$. Using a procedure similar to that of Love and Brownlee we have investigated the effects on the height and rate of production of ionization in a model meteor trail as we make significant changes in the mass, density and speed of the particle. The least significant effect is that due to a change in the density; a factor of two increase in particle density moves the whole ionization profile downward by about $2 \mathrm{~km}$.

For a given particle a change in speed of entry has a very significant effect on the height and degree of ionisation of the meteor trail. In Figure 1a is shown the predicted electron line densities for a stony meteoroid entering the atmosphere at 30 and $60 \mathrm{~km} / \mathrm{s}$. Doubling the velocity increases the value of the peak line density by a factor of 5.3 and raises the height of the trail by about 8 $\mathrm{km}$. Doubling the speed again to that of an interstellar meteoroid (say $120 \mathrm{~km} / \mathrm{s}$ ) has an even more dramatic effect on the height and intensity of the trail as is shown in Figure 1b. Thus interstellar meteors can be expected to produce very bright trails at heights above $105 \mathrm{~km}$, and possibly occurring as high as $135 \mathrm{~km}$.

Lebedinets (1991) has pointed out that meteoroids of low ablation temperature (say $500^{\circ}-$ $1,000^{\circ} \mathrm{K}$ ) may be very significant contributors to meteor trails occurring at heights above $110 \mathrm{~km}$. To test this suggestion we have modelled the case of an organic polymer (CHON-type particle) of density $\sim 1 \mathrm{gm} / \mathrm{cc}$, that ablates at a temperature of about $500^{\circ} \mathrm{K}$ (cf. $2000^{\circ} \mathrm{K}$ for a stony particle). The result is shown in Figure 2, where it is evident that such a particle with an incoming speed of $60 \mathrm{~km} / \mathrm{s}$ will produce ionization at heights between 120 and $170 \mathrm{~km}$, although the peak ionization is about one third of that for a stony particle of the same mass. It is conjectured that the ionization detected by the EISCAT system was deposited by a particle of this type.

CHON-type particles probably contain only a small fraction of the metallic atoms $\mathrm{Fe}, \mathrm{Na}, \mathrm{Mg}$, $\mathrm{Ca}$, which are the main contributors to the optical emission from meteor trails formed by stony particles. Thus it is not surprising that optical studies have not revealed evidence of moteors formed by CHON-particles. 


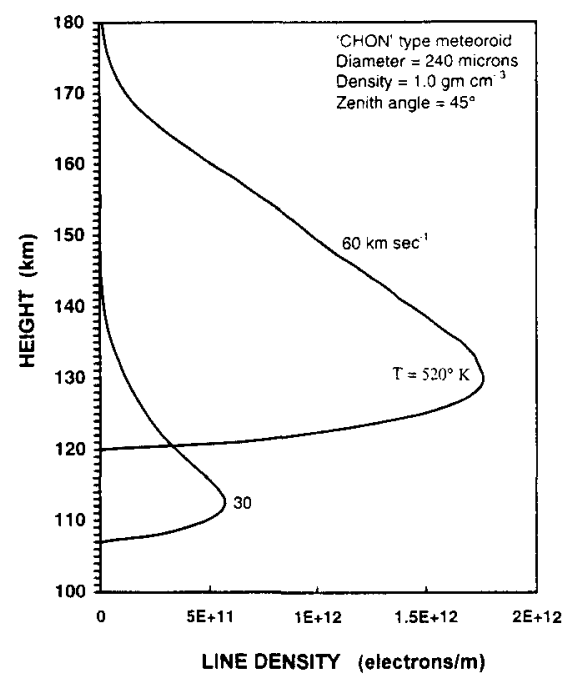

Figure 2. Effect of lowering the ablation temperature on the ionisation profile of a meteoroid.

\section{Velocity distribution of meteoroids}

The amount of ionisation produced by an ablating meteoroid is strongly dependent on the meteoroid speed as can be inferred from Figure 1. A survey by Bronshten (1983) of laboratory studies of the ionization coefficients of atoms that typify chondritic meteoroids indicates that if the mass and speed of a meteoroid are $m$ and $v$, the maximum electron line density has a mass-velocity dependence of the form $m v^{b}$, where $b=3.75$. The number of meteoroids, $N$, greater than a given mass $m_{\circ}$, is usually expressed by the relation

$$
N\left(m>m_{\circ}\right) \propto m_{\circ}^{-\alpha}
$$

where $\alpha$ is the cumulative mass index. Taylor (1995) has discussed the value of $\alpha$ and concludes that for the mass range of meteoroids associated with radar meteors, the most likely value for the mass index is 1.1 . Whence, to normalise the velocity distribution to a constant mass we apply a weight of the form $\left(v_{o} / v\right)^{\alpha b}$, where $\alpha b=4.13$. It is usual to choose $v_{o}$ as $30 \mathrm{~km} / \mathrm{s}$.

The most comprehensive set of observations of meteors formed by small meteoroids with a median mass of $10^{-4} \mathrm{gm}$ observed under nearly uniform conditions during one year, was obtained during the 1968-69 Harvard Radio Meteor Project. The velocity distribution of 14,220 meteoroids corrected for the effects of velocity bias on the meteoric ionisation and the radar detectability of the meteor trails, was published by Southworth and Sekanina (1973), and has been used extensively by subsequent workers. Recently Taylor (1995) has shown that a typographical error in the computer code used by Southworth and Sekanina caused the flux of high speed meteoroids to be underestimated by a factor of about 100. Taylor's initial revision of the meteoroid velocity distribution is shown in Figure 3 as the full line. Subsequently, the effects on the velocity distribution of the initial radius of the meteor trail and the diffusion of the ionisation were included, based on the model ionisation profiles discussed above (Elford et al., 1997 ). The new velocity distribution is shown in Figure 3 as filled circles.

\section{Distribution of meteor radiants and orbits}

The distribution of the radiants of meteors detected by the Harvard Radio Meteor Project is shown in Figure 4, where a weight has been applied to each observed radiant for the various selection effects described above. A correction for the flux enhancement due to the Earth's gravity has also been incorporated. Incomplete coverage of the southern-ecliptic hemisphere necessitates the assumption 


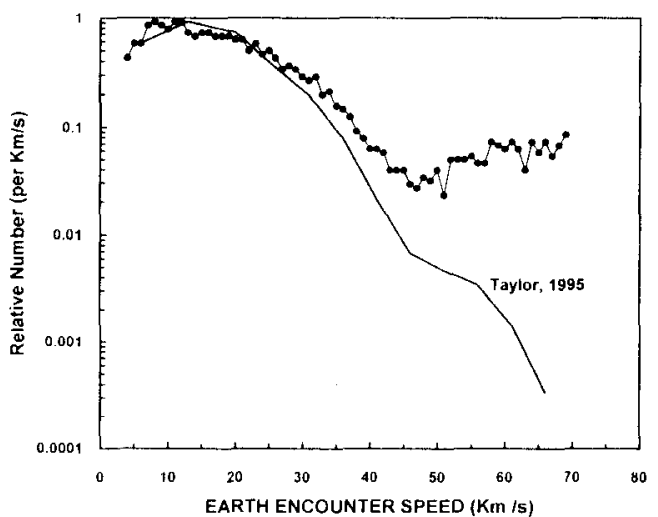

Figure 3. The speed distribution of meteoroids of median mass $10^{-4} \mathrm{gm}$ encountering the Earth, derived from meteors observed during the Harvard Radio Meteor Project 1968-69. The continuous line is Taylor's improved correction for the effect of velocity on ionisation production during ablation. The filled circles include the effect of diffusion on the radar detectability of a meteor trail.

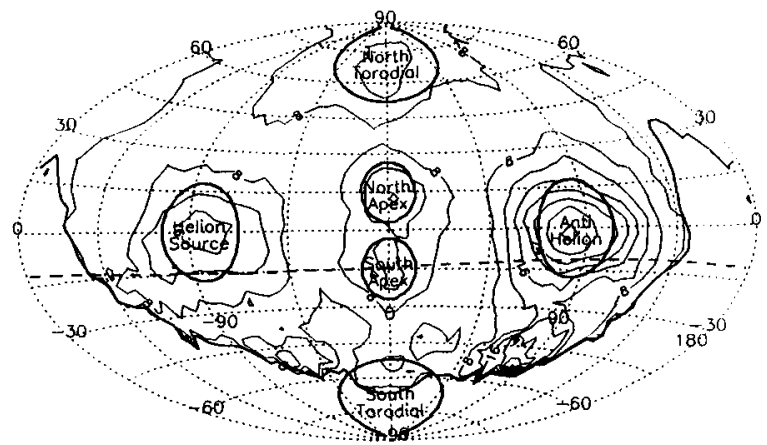

Figure 4. The radiant distribution of meteoroids with mass $>10^{-4} \mathrm{gm}$ encountering the Earth. The coordinate frame places the Earth at the centre, the solar (Helion) direction at a longitude of $-90^{\circ}$, and the north ecliptic pole at $+90^{\circ}$. The distribution is based on radar observations (see text) and has been corrected for selection effects associated with the radar detectability of meteors and the atmosphere. The heavy line is the limit of observation of the radar, and radiants south of the dashed line were observed for less than $10 \%$ of the time spent observing the region of the celestial north pole. The six radiant sources are overlaid with the one sigma radii appropriate to each source.

that the radiant distribution is symmetric about the ecliptic plane. Elford and Taylor (1997) have shown that linearly-polarised meteor echoes observed during the day may be significantly attenuated due to the effect of Faraday rotation. This is almost certainly the reason that the helion source in Figure 4 is of much lower strength than the anti-helion source, for one would expect to encounter as many metcoroids on highly elliptical low inclination orbits moving toward the vicinity of the Sun (anti-helion source) as there are moving away from the vicinity of the Sun (helion source). As we are uncertain of the value of the Faraday bias, we have not used the data described as the helion source but have assumed that the strength of this source is the same as that of the anti-helion source, and further that the distribution is symmetrical about the plane orthogonal to the plane of the ecliptic and passing through the apex and the poles.

Of the six major sources identified in Figure 4, the major ones are the helion and anti-helion contributed by metcoroids in prograde orbits, and the pair of apex sources contributed by meteoroids in retrograde orbits. The encounter speeds of 'helion/antihelion meteoroids' range between 10 and $40 \mathrm{~km} / \mathrm{s}$ with a median value of $20 \mathrm{~km} / \mathrm{s}$, while the encounter speeds of the 'apex meteoroids' are $50-70 \mathrm{~km} / \mathrm{s}$ with a median value of about $60 \mathrm{~km} / \mathrm{s}$.

The distribution in space of the orbits that contribute to the data presented in Figure 4 is best 

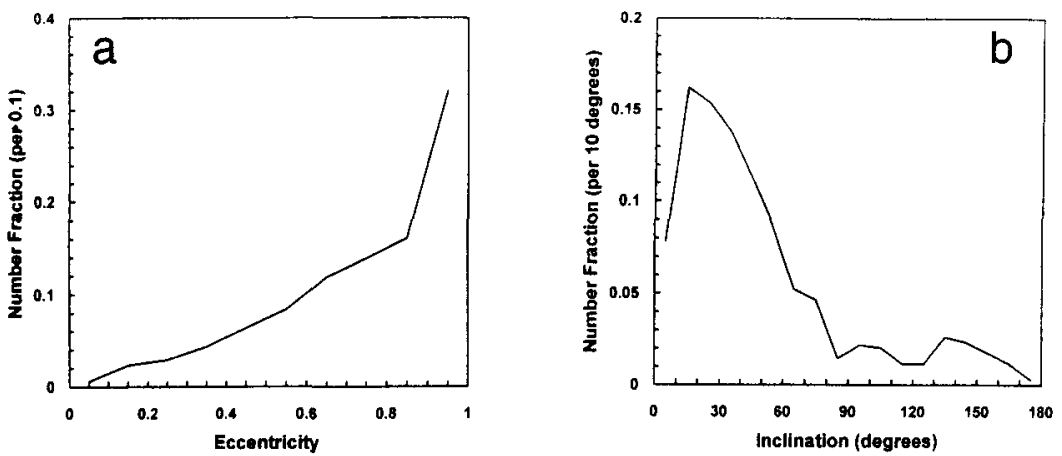

Figure 5. The distribution of the inclinations of the orbits of meteoroids contributing to the radiant distribution in Figure 4. Each orbit is weighted to account for the probability of a particle in the particular orbit colliding with the Earth. About $15 \%$ of the meteoroids move in retrograde orbits.

described in terms of the distributions of the orbital elements. In presenting such distributions it is necessary to take into account the probability of a particle in a particular orbit undergoing a collision with the Earth. This is achieved by weighting the data according to a procedure described by Kessler (1981).

The distribution of the eccentricities of the orbits is shown in Figure 5a. The majority of the orbits have eccentricities exceeding 0.4, and as the Poynting-Robertson effect on the motion of particles is to decrease the eccentricity of their orbits, there will be a tendency for points on this diagram to move from right to left over time.

The distribution of inclinations is shown in Figure 5b. The mean inclination is close to $40^{\circ}$. There is also a significant number of orbits with inclinations exceeding $90^{\circ}$; in fact the results imply that about $15 \%$ of meteoroids with masses $>10^{-4} \mathrm{gm}$ (diam $>200$ microns) move in retrograde orbits. Both distributions given here are very different than those derived by Southworth and Sekanina (1973) who used an incorrect velocity bias, an underestimate of the effect of diffusion, and took no account of the effect of Faraday rotation.

\section{Interstellar Meteors}

A small but significant fraction of meteors detected by the AMOR radar in New Zealand have velocities in excess of $100 \mathrm{~km} / \mathrm{s}$, implying that they are of interstellar origin. From the set of 1508 such meteors collected in 1990 and 1991, with a mean speed of $164 \mathrm{~km} / \mathrm{s}$, Taylor et al. (1996) have used the variation in flux throughout the year to identify two discrete sources, as shown in Figure 6. One source (A) is associated with nearby A-type stars, and the other (B) with the Sun's

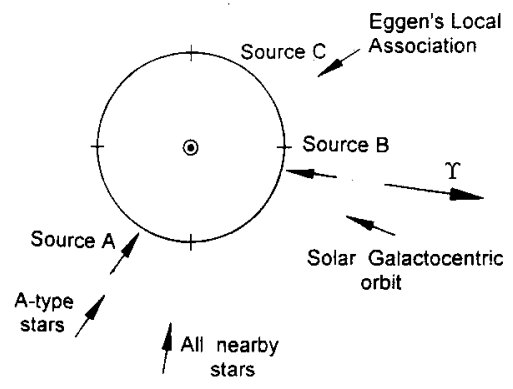

Figure 6. Possible sources of interplanetary meteoroids, projected on to the ecliptic. The symbol $\gamma$ shows the direction of the vernal equinox. Source $A$ is plausibly associated with the motion of the Solar System relative to the nearby A-type stars; source B is nearly aligned to the Sun's Galactocentric orbit; source C is in the direction of Eggen's local association of young star clusters and B-type stars. 

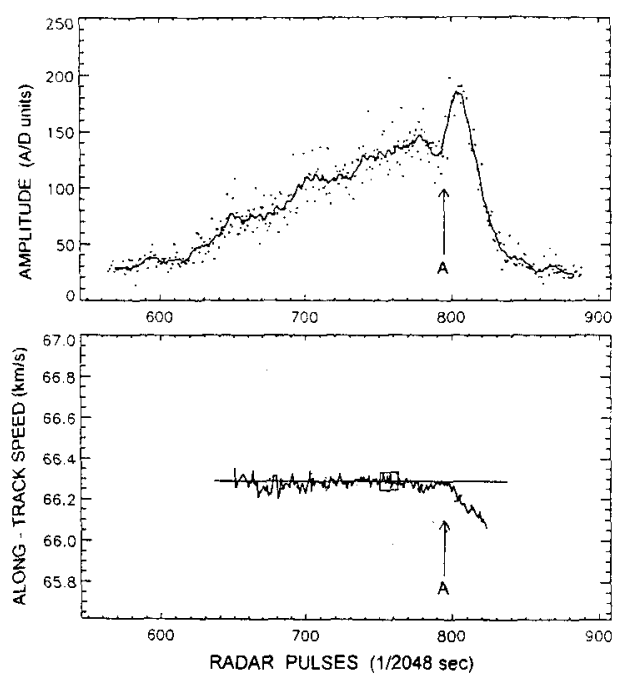

Figure 7. Amplitude of the meteor radar echo (top), and the speed of the ablating meteoroid determined from a 'head echo' observed using a narrow beam VHF radar. The meteoroid entered the radar beam at $21^{\circ}$ to the beam axis and during the time interval 600-750 units moved at constant speed. The particle then underwent a catastrophic event at $A$ and burnt out. The associated 'flare' in amplitude and the large deceleration suggests major fragmentation into many particles.

motion about the Galactic Centre. The data also suggest the presence of a third source (C), possibly associated with local B-type stars and young star clusters (Eggen's local association). The size of the interstellar particles producing these high velocity meteor trails is estimated to be in the range $15-40 \mu \mathrm{m}$.

\section{Head echoes}

Until recently it was generally accepted that radar detection of meteors was limited to those situations where the trail is orthogonal to the line of sight to the radar station. With this geometry, the strength of the echo is enhanced by the coherent scattering from the ionisation in a length of the trail about a Fresnel zone in extent. With the advent of narrow beam radars operating at VHF and UHF a new form of scattering has been observed from a small region near the head of a trail. A characteristic of this type of observation is the sequential occurrence of echoes in a number of range bins as the head of the trail advances down the radar beam, at angles up to $45^{\circ}$ from the beam axis.

Zhou et al. (1995) have reported that this type of meteor head echo has been observed with the $430 \mathrm{MHz}$ Arecibo incoherent scatter radar, and that a systematic study of such echoes was carried out on 'World Days' in 1991 and 1992. The echoes were observed over a height range of 80 to 120 $\mathrm{km}$. The observations are consistent with general view that the meteors with the greatest heights are produced by meteoroids with the highest speeds.

Narrow-beam $\left(2-3^{\circ}\right)$ VHF radars have also revealed the presence of 'down the beam' head echoes (Taylor et al. 1996). The time intervals between the appearance of the echoes in subsequent range bins give the line of sight velocity of the meteoroid. A better spced determination can be derived from the phase changes between the returned pulses. Although the meteoroid moves a distance equivalent to many wavelengths between radar pulses, the integer number of phase cycles can be determined by comparison with the line of sight velocity. The total phase-time record then gives the speed of the meteoroid along its trajectory, and also the angle between the trajectory and the axis of the radar beam. The observations give velocities of remarkable precision and make possible measurements of deceleration. Further, by sampling the amplitude of the echo in subsecquent range bins an amplitude-time profile of the echo can be determined and related to the distribution of ionisation along the trail. An example of such an observation is shown in Figure 7, where the 
meteor shows a terminal flare, probably due to severe fragmentation (at A). The fragmentation is also associated with a rapid change in speed (deceleration) during the final stages of the echo. Prior to the fragmentation the speed of the meteoroid was $66.3 \pm 0.05 \mathrm{~km} / \mathrm{s}$. The meteoroid crossed the radar beam at an angle of $21^{\circ}$ to the beam axis.

In cases where a meteoroid undergoes gross fragmentation during ablation into two parts of relatively similar mass the resultant trails will give rise to two radio echoes that beat together. Such head echoes are not uncommon and further studies of the amplitude characteristics and the associated deceleration measurements of 'head echoes' can be expected to contribute to an improved understanding of the physical structure of meteoroids.

\section{References}

Bronshten, V.A. 1983. Physics of meteoric phenomena, Reidel, Dordrecht.

Elford, W.G., and Taylor, A.D. 1997. Measurements of Faraday rotation of radar meteor echoes for the modelling of electron densities in the lower ionosphere, J. Atmos. Solar-Terr. Phys., 59, 1021-1024.

Elford, W.G., Steel, D.I., and Taylor, A.D. 1997. Implications for meteoroid chemistry from the height distribution of radar meteors, Adv. Space Sci., in press.

Kessler, D.J. 1981. Derivation of the collision probability between orbiting objects: the lifetimes of Jupiter's outer moons, Icarus, 48, 39-48.

Lebedinets, V.N. 1991. Organic particles of cometary dust in space, Adv. Space Res., 11(12), 149-153.

Öpik, E.J. 1958. Physics of Meteor Flight in the Atmosphere, Interscience Publishers, New York.

Love, S.G., and Brownlee, D.E. 1991. Heating and thermal transformation of micrometeoroids entering the Earth's atmosphere, Icarus, 89, 26-43.

Pellinen-Wannberg, A., and Wannberg, G. 1996. Enhanced ion-acoustic echoes from meteor trails, J. Atmos. Terr. Phys., 58, 495-506.

Southworth, R.B., and Sekanina, Z. 1973. Physical and dynamical studies of meteors, NASA Contractor Report CR-2316, Washington DC, pp 106.

Steel, D.I., and Elford, W.G. 1991. The height distribution of radio meteors: comparison of observations at different frequencies on the basis of standard echo theory, J. Atmos. Terr. Phys., 53, 409-417.

Taylor, A.D. 1995. The Harvard Radio Meteor Project meteor velocity distribution reappraised, Icorus, 116, 154-158.

Taylor, A.D., Cervera, M.A., Elford, W.G., and Steel, D.I. 1996. A new technique for radar meteor speed determination: inter-pulse phase changes from head echoes, in Physics, Chemistry, and Dynamics of Interplanetary Dust, B.A.S. Gustafson and M.S. Hanner, eds., ASP Conference Series, Vol 104, 75-78.

Zhou, Q., Tepley, C.A., and Sulzer, M.P. 1995. Meteor observations by the Arecibo $430 \mathrm{MHz}$ incoherent scatter radar - I. Results from time-integrated observations, J. Aimos. Terr. Phys., 57, 421-431. 